\title{
Upper Airway Resistance Syndrome
}

\author{
Abha Pandey ${ }^{1}$, Bhupendra Vala ${ }^{2}$, Miti Shah ${ }^{3}$ \\ International Journal of Head and Neck Surgery (2019): 10.5005/jp-journals-10001-1364
}

\section{Case Description}

A 35 years female with BMI of $23 \mathrm{~kg} / \mathrm{m}^{2}$, came with complaints of excessive daytime fatigability and sleepiness. Her husband gave history of snoring while sleep. She suffered with depression and had excessive sweating issues. Her limited channel study was normal. Complete overnight polysomnography showed lowest $\mathrm{SpO}_{2}$ of 92\%, AHI-2.5/hr, RDI-15.5/hr with RERAs-13/hr. No positional variation noted in $\mathrm{AHI}$.

Upper airway resistance syndrome (UARS) was first recognized in children in 1982, though the term UARS was not used until 1993, when adult cases were reported for the first time. ${ }^{1}$ The term UARS was devised to delineate a group of individuals who did not meet the criteria for obstructive sleep apnea (OSA) and therefore were not diagnosed or not treated, despite severe impairment. ${ }^{2}$ Pioneering work of Guilleminault and others in past three decades paved the way for UARS in the broader umbrella of disorders called sleep-disordered breathing (SDB) (Fig. 1). UARS is characterized by frequent arousals in response to increased respiratory effort as a result of upper airway narrowing without overt apnea or hypopnea. Global prevalence is around $10-15 \%$ when definition is applied to adults who suffer from snoring and excessive daytime somnolence (EDS).

The existence of UARS is still a matter of controversy with no clear-cut standardized diagnostic criteria. The lack of education about UARS in the medical community, and also the controversies related to its position within SDB results in health practitioners accepting the decisions from medical insurance companies and eventually no specific treatment is provided to UARS subjects.

\section{Pathophysiology}

The pharyngeal collapse depends on the balance between collapsible factors like UA narrowing, pharyngeal collapsibility and protective factors like UA-dilating muscle activity and the protective pharyngeal reflexes (Fig. 2). Patients with UARS exhibit typical craniofacial abnormalities (Fig. 3) leading to size reduction of the pharynx.
${ }^{1-3}$ Department of Respiratory Diseases, Lilavati Hospital and Research Center, Mumbai, Maharashtra, India

Corresponding Author: Abha Pandey, Department of Respiratory Diseases, Lilavati Hospital and Research Center, Mumbai, Maharashtra, India, e-mail: abhapandey85@gmail.com

How to cite this article: Pandey A, Vala B, Shah M. Upper Airway Resistance Syndrome. Int J Head Neck Surg 2019;10(1):18-21.

Source of support: Nil

Conflict of interest: None

Dematties et al. ${ }^{4}$ and Guilleminault et al. ${ }^{5}$ showed that UA sensitivity in UARS subjects is close to normal subjects. Due to unimpaired UA sensitivity, these patients have intact ability to activate the protective reflexes. They rather have very high levels of dilator muscle activity in response and lower arousal threshold making arousals frequent and impactful.

\section{Clinical Presentation}

Typically, patients with UARS are less obese, heavy snorers with excessive daytime sleepiness (Table 1). UARS patients do not have severe oxygen desaturation during sleep. Few patients also present with functional somatic syndrome, a form of complex presentation due to presence of psychological factors (depression), neurologic factors (increased pain sensitivity), hormonal factors (orthostatic hypotension and alterations in hypothalamo-pituitary-adrenal axis) and sleep-related factors (frequent arousals and alpha frequency intrusion into sleep). ${ }^{8}$ UARS generally remains stable over time. However, it may progress to OSA if there is an increase in body mass index (BMI) of patient.

\section{Cardiovascular Sequelae}

UARS has known to cause cardiovascular and metabolic consequences, attributed to snoring, increased respiratory efforts throughout the night and sleep fragmentation. Lee et al. ${ }^{9}$ in an observational cohort of 101 snorers and non-snorers with mild nonhypoxic OSAS, noted that heavy snoring ( $>50 \%$ night snoring) was

\begin{tabular}{|c|c|c|c|c|c|}
\hline \multicolumn{6}{|c|}{ Spectrum of sleep-related breathing disorders } \\
\hline & Snoring & & & Obstructive sleep apnea & \\
\hline $\begin{array}{l}\text { Normal } \\
\text { breathing }\end{array}$ & $\begin{array}{l}\text { Occasional } \\
\text { snoring } \\
\text { No disease }\end{array}$ & $\begin{array}{l}\text { Regular } \\
\text { snoring }\end{array}$ & $\begin{array}{l}\text { Upper airway } \\
\text { resistance } \\
\text { syndrome }\end{array}$ & $\begin{array}{cc}\text { Mild } & \text { Moderate } \\
\text { sleep apnea } & \text { sleep apnea } \\
\text { Disease }\end{array}$ & $\begin{array}{c}\text { Severe } \\
\text { sleep apnea }\end{array}$ \\
\hline
\end{tabular}

Fig. 1: Spectrum of SDB

(O)The Author(s). 2019 Open Access This article is distributed under the terms of the Creative Commons Attribution 4.0 International License (https://creativecommons. org/licenses/by-nc/4.0/), which permits unrestricted use, distribution, and non-commercial reproduction in any medium, provided you give appropriate credit to the original author(s) and the source, provide a link to the Creative Commons license, and indicate if changes were made. The Creative Commons Public Domain Dedication waiver (http://creativecommons.org/publicdomain/zero/1.0/) applies to the data made available in this article, unless otherwise stated. 


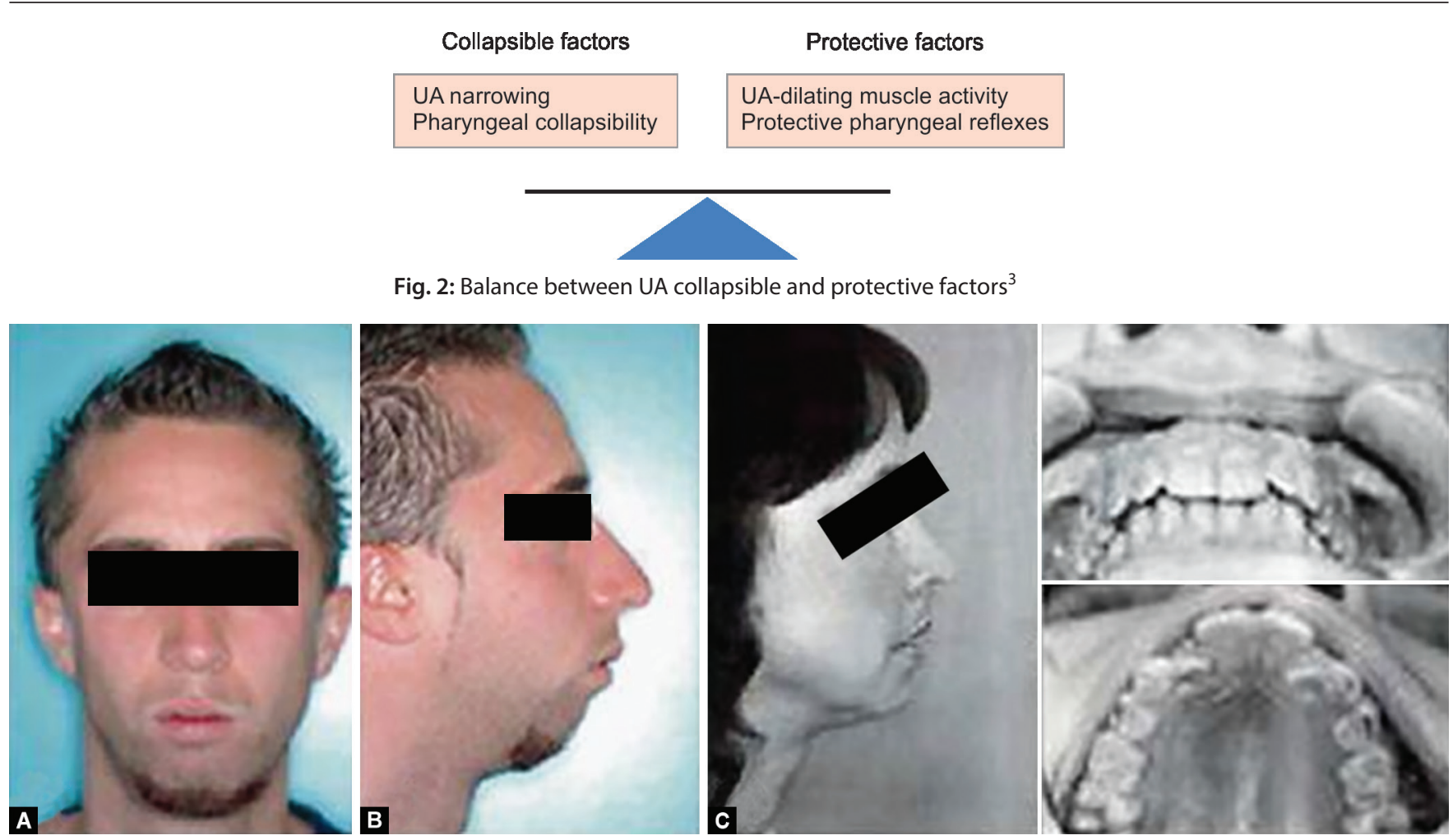

Figs 3A to C: Typical craniofacial features of UARS. (A and B) Long face syndrome with a short and narrow chin and with retrognathic chin; (C) Dental overjet and ogival hard palate in UARS. Courtesy: Dallas Center for Sleep Disorders.

Table 1: Clinical presentation of UARS

\begin{tabular}{ll}
\hline Symptoms & Signs $^{6}$ (shown in figure) \\
\hline - Snoring & Classical long face syndrome \\
- Nocturia/nocturnal awak- & with short and narrow chin and \\
ening/Sleep inefficiency & reduced mouth opening \\
- EDS & - Presence of classical 'Click' and \\
- Daytime fatigue & subluxation while opening \\
- Headache/vasomotor & Temporomandibular joint as \\
Rhinitis/Irritable bowel & evidenced by palpation \\
syndrome & Mandible in back position and \\
- Decreased performance/ & palate is high and narrow \\
Anxiety/depression & Low resting arterial blood pres- \\
& sure or orthostatic intolerance \\
\hline
\end{tabular}

an independent risk factor for carotid atherosclerosis. Convincingly, the prevalence of carotid atherosclerosis was found to increase progressively with severity of snoring. In UARS, the second key mechanism responsible for cardiovascular consequences is the occurrence of repetitive microarousals evident in the form of Respiratory effort-related arousals (RERA) episodes and consequent rise in airway resistance which in turn leads to repetitive increase in blood pressure in these patients. ${ }^{10}$ Therefore, UARS-induced sleep fragmentation and prolonged episodes of inspiratory flow limitation might lead to a chronic increase in sympathetic outflow that could explain the secondary occurrence of cardiovascular and metabolic consequences.

\section{Diagnosis}

Diagnosis of UARS depends upon the presence of supportive clinical presentation as explained above, accompanied with following positive findings on diagnostic evaluation.
- Increased respiratory effort on esophageal pressure monitoring (Gold standard). Three abnormal patterns are seen:

1. Pes crescendo

2. Sustained continuous respiratory effort

3. Pes reversal

- Polysomnography (PSG) findings:

- RERA event which is defined as sequence of breaths lasting for atleast 10 seconds and characterized by series of respiratory cycles of increasing/decreasing effort or flattening, recorded by nasal manometry and resulting in arousal, not defined by apnea or hypopnea.

- Normal AHI, i.e., no significant apnea or hypopnea

- No significant oxygen desaturation

- Air flow limitation evident as flattening of normal bell-shape curve of normal breath, with a drop in the amplitude of the curve by $2-29 \%$ compared to normal breaths immediately preceding.

In our case patient is young with normal BMI with suggestive symptoms of sleep disordered breathing and autonomic features. Her PSG has distinct RERAs but in significant AHI with normal oxygen levels. Thus, classical for UARS. UARS despite being on the same spectrum as SDB, has many contrasting features when compared to OSA as shown in Table 2.

\section{TREATMENT}

Treatment of UARS should be given due importance in view of its established consequences in form of cardiovascular and metabolic compromise. As snoring has been a major risk factor for the same, this domain can be handled with surgical intervention promisingly than other disease of SDB. ${ }^{11}$ 
Upper Airway Resistance Syndrome

Table 2: Comparison of features in UARS and OSAS

\begin{tabular}{|c|c|c|}
\hline Parameters & UARS & OSAS \\
\hline Age & All ages & $\begin{array}{l}\text { Children } \\
\text { Male }>40 \text { years } \\
\text { Female after Menopause }\end{array}$ \\
\hline M:F ratio & $1: 1$ & $2-3: 1$ \\
\hline Sleep onset & Insomnia & Fast \\
\hline Snoring & Common & Almost always \\
\hline Apnea & No & Common \\
\hline Daytime symptoms & Tiredness and fatigue & Sleepiness \\
\hline Body habitus & Slim or normal & Commonly Obese \\
\hline Somatic dysfunction & $\begin{array}{l}\text { Fibromyalgia } \\
\text { Chronic pain and headache }\end{array}$ & Rare \\
\hline Orthostatic symptoms & $\begin{array}{l}\text { Cold hands/feet } \\
\text { Fainting and dizziness }\end{array}$ & Rare \\
\hline Blood pressure & Low or normal & High \\
\hline Neck circumference & Normal & Large \\
\hline
\end{tabular}

Adapted from Guilleminault et al. Upper airway resistance syndrome-one decade later. Curr Opin Pulm Med 2004

- Continuous positive airway pressure (CPAP) therapy-lt is the mainstay of treatment though the patients have difficulty in tolerating CPAP and which is better evaluated in various studies. ${ }^{12,13}$

- Surgery-Surgical modality is helpful in patients who either do not tolerate CPAP or are not willing to adhere to CPAP. The choice of surgical procedure is usually based on the site of upper airway obstruction, with the understanding that multiple levels of increased airway resistance or collapse may exist.

- Uvulopalatopharyngoplasty (UPPP) and laser-assisted Uvulopalatopharyngoplasty (LAUP) are the most commonly performed surgeries for UARS, with good success rates; LAUP can be more cost-effective approach.

- A multilevel, pharyngeal surgical approach consisting of UPPP, mandibular osteotomy with genioglossus advancement (GA), and Hyoid myotomy with advancement (HM) proposed by Riley et al..$^{14,15}$ They reported a success rate of $60-65 \%$ postoperatively and a result equivalent to CPAP.

- Septoplasty with bilateral inferior turbinate resection should be reserved as an adjunct to pharyngeal surgery or to improve the tolerance of CPAP.

- Orthodontic approaches-Mandibular advancement devices and rapid maxillary distractionare easily performed in children and teenagers. These devices act by repositioning the lower jaw forward and reduce upper airway resistance.

- Oral Appliances $(O A)$ - these are the devices inserted into the mouth to modify the position of tongue and mandible, relieving obstruction during sleep. These devices have shown to improve snoring, transient arousals and EDS. ${ }^{16}$

- Cognitive behavioral therapy (CBT) in conjunction to CPAP in patients with chronic insomnia or psychosomatic symptoms can be helpful.

- Hormonal therapy-as an adjunct to CPAP and surgical modality is also helpful as shown in some studies, especially in menopausal women. ${ }^{13}$

UARS is a structural problem caused by smaller-than-normal jaw structures, leading to narrowed air-passages which eventually results in the pathophysiological changes and clinical features.
Eventually some proportion of patients will either not tolerate or adhere to CPAP therapy.

The two most common reasons for non-compliance with CPAP are obstruction within the nose and pharynx and lack of subjective improvement with treatment. Studies showed that CPAP did not improve sleep efficiency and sleep stages in these patients..$^{13}$ UARS patients do not tolerate CPAP very well, due to highly sensitive upper airway unlike OSAS patients where there is diminished upper airway muscle tone and neurology, as seen by Guilleminault et al in a long term outcome study in UARS subjects. ${ }^{17}$

\section{Pearls of Wisdom}

- UARS is a separate established entity which needs early diagnosis with level 1 study

- Functional Somatic syndrome with EDS and snoring should raise suspicion for UARS

- UARS needs treatment in view of cardiovascular consequences

- Close monitoring is needed in view of possibility of incipient OSA in some

- Surgical intervention has a role in UARS

\section{References}

1. Guilleminault $\mathrm{C} 1$, Stoohs $\mathrm{R}$, et al. A cause of daytime sleepiness: the upper airway resistance syndrome. Chest. 1993;104(3):781-787.

2. Diagnostic classification steering committee. The internationa classification of sleep disorders: diagnostic and coding manual. Rochester. Minn: American Sleep Disorders Association. 1990.

3. Dempsey JA, Veasey SC, et al. Pathophysiology of sleep apnea. Physiol Rev 2010;90:47-112

4. Dematteis $M$, Levy $P$, et al. A simple procedure for measuring pharyngeal sensitivity: a contribution to the diagnosis of sleep apnoea. Thorax 2005;60:418-426.

5. Guilleminault C, Li K, et al. Two-point palatal discrimination in patients with upper airway resistance syndrome, obstructive sleep apnea syndrome, and normal control subjects. Chest 2002;122:866-870.

6. Guilleminault C, Black JE, et al. High (or abnormal) upper airway resistance (in French). Rev Mal Respir 1999;16:173-180.

7. Guilleminault C, Faul JL, et al. Sleep disordered breathing and hypotension. Am J Respir Crit Care Med 2001;164:1242-1247.

8. Goldenberg DL. Fibromyalgia syndrome a decade later: what have we learned? Arch Intern Med 1999;159:777-785. 
9. Lee $\mathrm{SA}$, Amis TC, et al. Heavy snoring as a cause of carotid artery atherosclerosis. Sleep 2008;31:1207-1213.

10. Guilleminault C, Stoohs R, et al. Upper airway resistance syndrome, nocturnal blood pressure monitoring, and borderline hypertension. Chest 1996;109:901-908.

11. Luciana BM de Godoy, Luciana O Palombini, et al. Treatment of upper airway resistance syndrome in adults:Where do we stand Sleep Sci. 2015 Jan-Mar;8(1):42-48.

12. Ong $\mathrm{KC}$, Cheng $\mathrm{PP}$, et al. Upper airway resistance syndrome-report of three cases. Ann Acad Med Singapore 2000;29:242-245.

13. Watanabe T, Mikami A, et al. Clinical characteristics of upper airway resistance syndrome. Psychiatry ClinNeurosci 1999;53:331-333.
14. Riley RW, Powell NB, et al. Obstructive sleep apnea and the hyoid: a revised surgical procedure. Otolaryngo1 Head Neck Surg 1994;111:717-721.

15. Riley RW, Powell NB, et al. Obstructive sleep apnea syndrome: a review of 306 consecutively treated surgical patients. Otolaryngol Head Neck Surg 1993;108.

16. Successful treatment of upper airway resistance syndrome with an oral appliance. Loube DI1, Andrada T, Shanmagum N, Singer MT. Sleep Breath. 1997 Dec;2(4):98-101.

17. Christian Guilleminault, Ceyda Kirisoglu, et al. Upper airway resistance syndrome: A long-term outcome study. J Psychiatr Res. 2006 Apr;40(3):273-279. 